\title{
The Huber theorem for non-compact conformally flat manifolds
}

\author{
Gilles Carron and Marc Herzlich
}

\begin{abstract}
It was proved in 1957 by Huber that any complete surface with integrable Gauss curvature is conformally equivalent to a compact surface with a finite number of points removed. Counterexamples show that the curvature assumption must necessarily be strengthened in order to get an analogous conclusion in higher dimensions. We show in this paper that any non compact Riemannian manifold with finite $L^{n / 2}$-norm of the Ricci curvature satisfies Huber-type conclusions if either it is a conformal domain with volume growth controlled from above in a compact Riemannian manifold or if it is conformally flat of dimension 4 and a natural Sobolev inequality together with a mild scalar curvature decay assumption hold. We also get partial results in other dimensions.
\end{abstract}

Mathematics Subject Classification (2000). 53C21, 58J60.

Keywords. Non compact Riemannian manifolds, Ricci curvature, conformal geometry, compactification, asymptotically locally euclidean manifolds.

\section{Introduction}

The main theme of this paper is the study of the geometry of non-compact manifolds with asymptotically zero Ricci curvature. In other words, we will consider throughout this paper complete Riemannian manifolds $(M, g)$ satisfying

$$
\int_{M}\left|\operatorname{Ric}_{g}\right|^{\frac{n}{2}}<\infty .
$$

Our goal is to investigate the consequences of assumption (1.1) on the asymptotic behaviour of the metric. We are mainly interested in possible generalizations in higher dimensions of the well-known and beautiful 1957's result by Huber [18]: every complete surface with integrable negative part of the Gauss curvature has integrable Gauss curvature and is conformally equivalent to a compact surface with a finite number of points removed. It is well known that any naive generalization of this result in higher dimensions is wrong. For instance, examples of manifolds with asymptotically non-negative curvature (in an integral sense) and infinite homotopy type (i.e. they are not equivalent to the interior of a compact 
manifold with boundary) are known [26]. Moreover, there exist as well complete Riemannian manifolds with finite volume, bounded curvature and infinite topological type. On the other hand, Abresch and later Kasue showed that some topological information may be available as soon as the curvature $K_{g}$ satisfies

$$
\int_{\mathbb{R}^{+}} \max \left\{\left|K_{g}(x)\right|, r(x) \geqslant \rho\right\} \rho d \rho<\infty
$$

where $r$ is the distance to a fixed point (see $[1,20]$ ). To obtain precise geometrical information, it is usually necessary to add an extra volume or diameter growth assumption (see for example [25, 31]). And the asymptotic geometry is known to be simple only when the whole curvature tensor decays at infinity in a very strong sense, e.g. if $K_{g}=\mathrm{O}\left(r^{-2-\varepsilon}\right)$ where $\varepsilon>0[4,15]$.

In order to obtain conformal information on the behaviour at infinity, our assumption (1.1) of finiteness of the $L^{n / 2}$-norm of Ricci curvature must then be strengthened a bit in order to stand in the middle of the two extremes. In this paper, we shall study two important special cases, each considered with natural extra assumptions.

In the first setting, the manifold is an arbitrary domain already embedded in a compact manifold and the metric is conformal to the "compact" metric. As expected, a Huber-type result follows if one adds control from above on the volume growth. This is the contents of our first main result (Theorem 2.1). As this case is rather special, it turns out that control on the full Ricci curvature is not necessary and some results are already available when one has only finiteness of the $L^{n / 2}$ norm of the scalar curvature (see Theorem 2.1 for details). Our result may be compared with a classical result by $\mathrm{K}$. Uhlenbeck: any Hermitian vector bundle on the euclidean ball $\mathbf{B}^{n}-\{0\}$ whose curvature is in $L^{n / 2}$ extends $W^{1, n}$ on the whole ball [27].

In the second, we release the assumption on topology (no compact manifold involved) and treat the case of conformally flat manifolds, which may be seen as the closest analogues of surfaces in higher dimensions. Our manifolds will satisfy finiteness of the $L^{n / 2}$-norm of Ricci curvature (1.1), together with an adequate Sobolev inequality:

$$
\mu_{n}(M, g)\left(\int_{M} u^{\frac{2 n}{n-2}}\right)^{1-\frac{2}{n}} \leqslant \int_{M}|d u|^{2}, \quad \forall u \in C_{0}^{\infty}(M),
$$

which is the extra hypothesis we choose for this case. For technical reasons, we also have to add some mild assumption on scalar curvature:

$$
\left.\mathrm{Scal}_{g} \in L^{\frac{n}{2}(1-\delta)} \cap L^{\frac{n}{2}(1+\delta)}, \quad \text { for some } \delta \in\right] 0,1[\text {. }
$$

We notice that the Sobolev assumption is automatically satisfied in the case of manifolds with positive Yamabe invariant together with finite $L^{n / 2}$-norm (1.1) of Ricci curvature. Things run smooth in dimension 4 and we prove our second main result (Theorem 5.7): under these assumptions, the manifold is conformally 
equivalent to a compact orbifold with singularities (in finite number) removed. As elementary examples show, it is impossible to rule out the orbifold-type singularities in dimension $n \geqslant 3$ as they may give rise, by sending conformally the singularity to infinity, to manifolds and metrics satisfying our conditions. In other dimensions, we are forced to strengthen the condition and to assume quadratic decay on Ricci curvature to get a result valid in any dimension, and this is our third main result (Theorem 5.9).

Our results should be compared with recent and independent results due to S. Y. A. Chang, J. Qing and P. Yang [8]. They obtained a similar compactification theorem for conformal complete metrics on domains in the round sphere $S^{4}$. However, their assumptions are different from ours. Their results use only finiteness of the Gauss-Bonnet-Chern integral rather than $L^{2}$-integrability of the Ricci curvature but they need to impose uniformly positive scalar curvature (which would be in our context a rather unnatural restriction). It should also be noticed that our results apply to different situations where no assumption on topology is made.

We conjecture that our result in the conformally flat case can be obtained without the extra condition on scalar curvature. In the same vein, it would be interesting to know whether the Sobolev inequality assumption is necessary. Whereas such an assumption is certainly needed (see Remark 5.8), it is possible that only a weaker form of it is enough to obtain a Huber-type conclusion. It follows however from section 2 that each manifold showing Huber's behaviour at infinity does satisfy the Sobolev inequality. Hence, it is not unreasonable to impose it in our assumptions. It would of course also be highly desirable to have an answer for non conformally flat manifolds. We intend to consider all these questions in a future work.

The structure of the paper is as follows.

In section 2 , we consider the simple case where the manifold is a domain in a compact manifold and the complete metric is already conformal to a smooth metric defined on the compact manifold (but no conformally flat assumption). The proofs rely on elementary potential theory together with analysis at infinity due to the first author [8].

In section 3, we present our first technical result. It shows that under the extra assumption on the behaviour of the scalar curvature, every complete manifold satisfying assumption (1.1) on Ricci can be endowed with another, conformally related and quasi-isometric, metric with vanishing scalar curvature around infinity, regardless of inner topology or geometry.

Section 4 is in a sense a digression from our main goal, but we found useful to include it. Here we show that any complete Riemannian manifold that already satisfies all our assumptions and the conclusion of Huber's theorem is necessarily conformally quasi-isometric to an asymptotically locally euclidean (ALE) manifold. This remark provides some information on the geometry near the punctures of the metrics studied in section 1: they are necessarily obtained by a stereographic 
conformal blow-up from the "compact" metric - in the terminology of $[13,22]$. It also aims at justifying our strategy in the next section, which reduces Huber's problem to finding ALE structures in the conformal class of the original metric.

Section 5 achieves the main goal of the paper. Building on work of S. Bando, A. Kasue and H. Nakajima [4], our second technical result proves that any complete conformally flat manifold, satisfying both (1.1) on Ricci and Sobolev and which is scalar flat around infinity, is asymptotically locally euclidean. It remains to apply our previous analysis: in dimension 4, the conformal metric found in section 3 is shown to retain condition (1.1) on its Ricci curvature. Hence it is asymptotically locally euclidean. In other dimensions, it is unclear whether this occurs, and we need to impose quadratic decay of the Ricci curvature. In both cases, we end with a conformally flat asymptotically locally euclidean manifold and the conclusion is ensured by previous work of the second author [17]. Moreover, the proof shows that everything in this section works equally well if our manifold is assumed to be conformally flat in a neighbourhood of infinity only.

\section{Domains in a compact manifold}

We consider here the geometry of domains contained in a compact manifold. The purpose of this section is to show that any domain $\Omega$ endowed with a complete metric which is conformally compact (in the sense that another conformally related metric extends smoothly over the boundary), having moreover asymptotically zero curvature in an integral sense and controlled volume growth, satisfies the conclusions of Huber's theorem: it is a compact manifold minus a finite set of points. More precisely, we prove the following:

Theorem 2.1. Let $\Omega$ be a domain of $\left(M, g_{0}\right)$, a compact Riemannian manifold of dimension $n>2$. Assume $\Omega$ is endowed with a complete Riemannian metric $g$ which is conformal to $g_{0}$. Suppose moreover that

$$
\text { - either the Ricci tensor of } g \text { is in } L^{\frac{n}{2}}(\Omega, g) \text { and } \operatorname{vol}_{g} B\left(x_{0}, r\right)=o\left(r^{n} \log ^{n-1} r\right)
$$
for some point $x_{0}$ in $\Omega$;

- or the positive part $\mathrm{Scal}_{+}$of the scalar curvature of $g$ is in $L^{\frac{n}{2}}(\Omega, g)$ and, for some point $x_{0}$ in $\Omega, \operatorname{vol}_{g} B\left(x_{0}, r\right)=O\left(r^{n}\right)$.

Then there is a finite set $\left\{p_{1}, \ldots, p_{k}\right\} \subset M$ such that

$$
\Omega=M-\left\{p_{1}, \ldots, p_{k}\right\} .
$$

The proof of the Theorem is divided into two steps: in the first one, we show that the Hausdorff dimension of $M-\Omega$ is zero; in the second, we show that $M-\Omega$ has a finite number of connected components. The first step relies on the following:

Proposition 2.2. Let $\Omega$ be a domain in $\left(M, g_{0}\right)$, a compact Riemannian manifold 
of dimension $n$. Assume that $\Omega$ is endowed with a complete Riemannian metric $g$ which is conformal to $g_{0}$. Assume that there is a point $x_{0} \in \Omega$ such that the geodesic ball (for the metric $g$ ) with center $x_{0}$ satisfies

$$
\operatorname{vol}_{g} B\left(x_{0}, r\right)=o\left(r^{n} \log ^{n-1} r\right), \quad \text { for } r \rightarrow \infty,
$$

then the $n$-capacity of $M-\Omega$ is zero, and the Hausdorff dimension of $M-\Omega=\partial \Omega$ is zero.

The proof of this proposition is reminiscent of the proof of Proposition 2.5 in Schoen and Yau's paper [24].

Proof of Proposition 2.2. We are going to prove that there is a sequence of bounded Lipschitz functions with compact support in $\Omega$, denoted $\left(f_{k}\right)_{k}$, such that

$$
\begin{aligned}
& \text { i) } \lim _{k \rightarrow \infty} \int_{\Omega}\left|d f_{k}\right|^{n}=0, \\
& \text { ii) } \lim _{k \rightarrow \infty} f_{k}=1 \text {, uniformly on the compact sets of } \Omega
\end{aligned}
$$

where the $L^{n}$-norm of the gradient is taken either with respect to the metric $g_{0}$ of $M$ or with respect to the metric $g$, as this integral is conformally invariant. We note moreover that the $L^{n}\left(M, g_{0}\right)$-limit of this sequence is the characteristic function of $\Omega$. We choose a function $f_{k}(x)=u_{k}(r)$, where $r$ is the distance function (for the metric $g$ ) to some fixed point $x_{0}$, and $u_{k}$ is defined as follows

$$
\begin{cases}u_{k}(r)=1 & \text { if } r \leqslant \sqrt{k} \\ u_{k}(r)=0 & \text { if } r \geqslant k \\ u_{k}(r)=\log (k / r) / \log (\sqrt{k}) & \text { if } \sqrt{k} \leqslant r \leqslant k\end{cases}
$$

As a result we have

$$
\int_{\Omega}\left|d f_{k}\right|_{g}^{n}(x) d \operatorname{vol}_{g}(x)=\int_{\sqrt{k}}^{k} \frac{1}{(\log \sqrt{k})^{n}} \frac{1}{r^{n}} d V(r),
$$

where $V(r)$ is the function $V(r)=\operatorname{vol} B\left(x_{0}, r\right)$; now integration by parts leads to

$$
\int_{\Omega}\left|d f_{k}\right|_{g}^{n}(x) d \operatorname{vol}_{g}(x)=\frac{V(k)}{k^{n}(\log \sqrt{k})^{n}}-\frac{V(\sqrt{k})}{(\sqrt{k})^{n}(\log \sqrt{k})^{n}}+n \int_{\sqrt{k}}^{k} \frac{V(r)}{(\log \sqrt{k})^{n}} \frac{d r}{r^{n+1}} .
$$

At the end we arrive to

$$
\int_{\Omega}\left|d f_{k}\right|_{g}^{n}(x) d \operatorname{vol}_{g}(x)=o(1) \quad \text { if } \quad k \longrightarrow \infty .
$$

This proves that the $n$-capacity of $M-\Omega$ is zero, so that the Hausdorff dimension of $\partial \Omega=M-\Omega$ is zero. 
The second step relies on the following Proposition

Proposition 2.3. Let $\Omega$ be a domain in $\left(M, g_{0}\right)$, a compact Riemannian manifold of dimension $n$. Assume that $\Omega$ is endowed with a complete Riemannian manifold $g$ is conformal to $g_{0}$. Assume that the positive part of scalar curvature of $(\Omega, g)$ satisfies that

$$
\int_{\Omega} \operatorname{Scal}_{+}^{\frac{n}{2}}(x) d \operatorname{vol}_{g}(x)<\infty
$$

then for a $\mu>0,(\Omega, g)$ satisfies the Sobolev inequality:

$$
\mu\left(\int_{\Omega} u^{\frac{2 n}{n-2}}\right)^{1-2 / n} \leqslant \int_{\Omega}|d u|^{2}, \quad \forall u \in C_{0}^{\infty}(\Omega) .
$$

Proof. According to [8, Proposition 2.5], we know that it is enough to show that the Sobolev inequality holds outside some compact set of $\Omega$, i.e. that we have, outside a compact $K$, the Sobolev inequality

$$
C\left(\int_{\Omega-K} u^{\frac{2 n}{n-2}}\right)^{1-2 / n} \leqslant \int_{\Omega-K}|d u|^{2}, \quad \forall u \in C_{0}^{\infty}(\Omega-K) .
$$

But our assumption on the scalar curvature of $g$ implies it is enough to show that outside a compact $K$ of $\Omega$ we have for any $u$ in $C_{0}^{\infty}(\Omega-K)$,

$$
C\left(\int_{\Omega-K} u^{\frac{2 n}{n-2}} d \operatorname{vol}_{g}\right)^{1-2 / n} \leqslant \int_{\Omega-K}\left(|d u|^{2}+\frac{n-2}{4(n-1)} \operatorname{Scal}_{g} u^{2}\right) d \operatorname{vol}_{g},
$$

As the matter of fact, choose another compact set $K^{\prime}$ containing $K$ such that

$$
\left(\int_{\Omega-K^{\prime}} \mathrm{Scal}_{+}^{n / 2}\right)^{2 / n} \leqslant \frac{n-1}{8(n-2)}
$$

then with (2.2) and the help of Hölder inequality, we have the Sobolev inequality on $\Omega-K^{\prime}$ for the constant $\mu=C / 2$. Now we have to prove that the Yamabe constant of $(\Omega-K, g)$ is positive. Here the Yamabe invariant of $(\Omega-K, g)$ is defined by

$$
Y(\Omega-K, g)=\inf _{u \in C_{0}^{\infty}(\Omega-K)}\left\{\int_{\Omega-K}|d u|^{2}+\frac{n-2}{4(n-1)} \operatorname{Scal}_{g} u^{2}, \quad\|u\|_{L^{2 n /(n-2)}}=1\right\} .
$$

The Yamabe invariant is a conformal invariant, hence it is enough to find a compact set in $\Omega$ such that $Y\left(\Omega-K, g_{0}\right)>0$. Let $L$ be the conformal Laplacian of $g_{0}$ :

$$
L=\Delta^{g_{0}}+\frac{n-2}{4(n-1)} \operatorname{Scal}_{g_{0}} .
$$

For any open set $O$ of $M$ of small volume, the first eigenvalue of the Laplace operator of $g_{0}$ on $O$ for the Dirichlet boundary condition is bounded from below 
by $C\left(\operatorname{vol}_{g_{0}} O\right)^{-2 / n}$ where $C$ is a geometrical constant of $\left(M, g_{0}\right)$. Thus, if we choose a compact $K$ such that the volume of $\Omega-K$ is small enough, the operator $L$ is coercive on $W_{0}^{1,2}(\Omega-K)$, that is to say there is a constant $\lambda>0$ such that

$$
\lambda\|u\|_{L^{2}}^{2} \leqslant \int_{\Omega-K} u L u d \operatorname{vol}_{g_{0}}, \forall u \in C_{0}^{\infty}(\Omega-K) .
$$

This implies that the topology of the Hilbert space $W_{0}^{1,2}(\Omega-K)$ is given by the quadratic form

$$
u \mapsto \int_{\Omega-K} u L u d \operatorname{vol}_{g_{0}}=\int_{\Omega-K}\left(|d u|^{2}+\frac{n-2}{4(n-1)} \operatorname{Scal}_{g_{0}} u^{2} d \operatorname{vol}_{g_{0}}\right) .
$$

Since $\Omega-K$ is relatively compact in $M$, the Sobolev space $W_{0}^{1,2}(\Omega-K)$ is embedded in $L^{2 n /(n-2)}(\Omega-K)$, and this embedding implies that the Yamabe constant of $\left(\Omega-K, g_{0}\right)$ is positive.

Remark 2.4. It should be noticed that the last two propositions imply that if a domain of a compact Riemannian manifold is endowed with a conformal metric with non-positive scalar curvature then the Sobolev inequality 2.1 holds; in particular the volume of geodesic balls satisfies a uniform euclidean-type lower bound. This has the consequence that, for example, the Riemannian product $\mathbb{R} \times \mathbf{T}^{n-1}$ cannot be conformally embedded in the flat torus $\mathbf{T}^{n}$.

Proof of Theorem 2.1. With either set of assumptions of the Theorem, Proposition 2.2 then tells us that the Hausdorff dimension of $\partial \Omega=M-\Omega$ is zero. Now, our assumption on the scalar curvature implies that we can apply the second Proposition: $(\Omega, g)$ satisfies the Sobolev inequality

$$
\mu\left(\int_{\Omega} u^{\frac{2 n}{n-2}}\right)^{1-2 / n} \leqslant \int_{\Omega}|d u|^{2}, \quad \forall u \in C_{0}^{\infty}(\Omega) .
$$

(i) We now turn to the case where $\mathrm{Scal}_{+}$is in $L^{\frac{n}{2}}$. Here [7, Proposition 2.4] shows that the Sobolev inequality implies a uniform lower bound on the volume of geodesic balls of $(\Omega, g)$

$$
\operatorname{vol}_{g} B(x, r) \geqslant\left(\frac{\mu}{2^{n+2}}\right)^{n / 2} r^{n}, \forall x \in \Omega, r>0 .
$$

This lower bound and the assumption on volume growth imply that $(\Omega, g)$ has a finite number of ends. As a matter of fact, let $x_{0}$ be a fixed point in $\Omega$ and let $R>0$. In each unbounded connected component $C$ of $\Omega-B\left(x_{0}, R\right)$ we can find a point $x_{C}$ at distance $2 R$ from $x_{0}$. The geodesic ball of radius $R$ around $x_{C}$ is in $C$ and in the ball $B\left(x_{0}, 3 R\right)$. Moreover, all these balls are disjoint. Thus the sum of the volumes of these geodesic balls is bounded from below by $\left(\frac{\mu}{2^{n+2}}\right)^{n / 2} R^{n}$ times the number of unbounded connected components, whereas this sum is also bounded from above by the volume of $B\left(x_{0}, 3 R\right)$, hence is bounded by $C^{\prime}\left(x_{0}\right) R^{n}$. 
These two inequalities show that the number of unbounded connected components of $\Omega-B\left(x_{0}, R\right)$ is no more than $C^{\prime}\left(x_{0}\right)\left(\frac{\mu}{2^{n+2}}\right)^{-n / 2}$. Since this bound is uniform with respect to $R,(\Omega, g)$ has a finite number of ends.

(ii) In case $\mathrm{Ric}_{g}$ is in $L^{\frac{n}{2}}$, one does not need any volume control to ensure finiteness of the number of ends since one may apply [8]: the number of ends is bounded by the dimension of the first reduced $L^{2}$-cohomology group, which is finite under our curvature assumption.

Considering now both cases together again, we get that $\partial \Omega$ has a finite number of connected components. Dimension theory [19] tells us that a connected set which is not a point has topological dimension greater than 1 . Since Hausdorff dimension is always greater than topological dimension, $\partial \Omega$ is a finite set.

For future reference, we note that a basic consequence of our arguments is:

Lemma 2.5. Let $\Omega$ be a domain of a compact Riemannian manifold $\left(M, g_{0}\right)$ of dimension $n>2$. Assume $\Omega$ is endowed with a complete Riemannian metric $g$ which is conformal to $g_{0}$, moreover assume that the volume of geodesic balls of $(M, g)$ is bounded from below uniformly:

$$
\exists C>0, \forall x \in M, \forall r \geq 1, \operatorname{vol}_{g} B(x, r) \geqslant C r^{n} .
$$

and that for a point $x_{0}$ in $(M, g)$ we have

$$
\operatorname{vol}_{g} B\left(x_{0}, r\right) \leqslant C^{\prime} r^{n}, \forall r \geqslant 1,
$$

then there is a finite set $\left\{p_{1}, \ldots, p_{k}\right\} \subset M$ such that

$$
\Omega=M-\left\{p_{1}, \ldots, p_{k}\right\} .
$$

Theorem 2.1 has an interesting application in the case of the sphere:

Corollary 2.6. Let $\Omega$ be a domain of the sphere $\left(\mathbf{S}^{n}, g_{0}\right)$ of dimension $n>2$, endowed with a complete Riemannian metric $g$ conformal to $g_{0}$ and satisfying either

$$
\int_{\Omega}\left|\operatorname{Ric}_{g}\right|^{\frac{n}{2}}(x) d \operatorname{vol}_{g}(x)<\infty \text { and } \operatorname{vol}_{g} B\left(x_{0}, r\right)=o\left(r^{n} \log ^{n-1} r\right)
$$

or

$$
\int_{\Omega}\left(\operatorname{Scal}_{+}\right)^{\frac{n}{2}}(x) d \operatorname{vol}_{g}(x)<\infty \quad \text { and } \operatorname{vol}_{g} B\left(x_{0}, r\right)=O\left(r^{n}\right) .
$$

Then there is a finite set $\left\{p_{1}, \ldots, p_{k}\right\} \subset \mathbf{S}^{n}$ such that $\Omega=\mathbf{S}^{n}-\left\{p_{1}, \ldots, p_{k}\right\}$.

Note that a much stronger result will be obtained in dimension 4 at the end of the paper (Corollary 5.13). 


\section{Scalar curvature uniformization of non-compact manifolds with asymptotically zero Ricci curvature}

Our second interest is in uniformization, by which we mean finding on such a manifold a best possible metric. The goal of the paper imposes us to restrict ourselves to conformal deformations only. Inspired by the well known Yamabe problem, we seek a metric with vanishing scalar curvature at infinity and which retains the asymptotic properties of the original metric. This is the only place where our extra assumption on scalar curvature is needed.

Theorem 3.1. If $\left(M^{n}, g\right)$ is a complete Riemannian manifold which satisfies the Sobolev inequality

$$
\mu_{n}(M, g)\left(\int_{M} u^{\frac{2 n}{n-2}}\right)^{1-2 / n} \leqslant \int_{M}|d u|^{2}, \quad \forall u \in C_{0}^{\infty}(M)
$$

and whose scalar curvature satisfies for a $\delta \in] 0,1[$,

$$
\mathrm{Scal}_{g} \in L^{\frac{n}{2}(1-\delta)} \cap L^{\frac{n}{2}(1+\delta)}
$$

then there is a bounded smooth function $\rho: M \rightarrow \mathbb{R}$ such that the complete metric $e^{2 \rho} g$ has vanishing scalar curvature outside some compact set.

Proof. In fact, we will find a function $u$ which is defined outside some compact set $K \subset M$ and solves the equation

$$
\Delta u+c_{n} \mathrm{Scal}_{g} u=-c_{n} \mathrm{Scal}_{g} \quad \text { outside } K
$$

where $c_{n}=(n-2) / 4(n-1)$; moreover $|u| \leqslant 1 / 2$ on $M-K$. Letting $v$ be the positive smooth function $v=1+u$ outside some bounded neighbourhood of $K$, the new metric $v^{\frac{4}{n-2}} g$ will have vanishing scalar curvature outside the compact set - recall the scalar curvature of the metric $v^{\frac{4}{n-2}} g$ is given by the formula

$$
c_{n} \mathrm{Scal}_{v^{\frac{4}{n-2}} g}=v^{-\frac{n+2}{n-2}}\left(\Delta v+c_{n} \operatorname{Scal}_{g} v\right) .
$$

The proof will be done in two steps. In the first step, we show that equation (3.1) has a solution $u \in L^{\frac{n}{2} \frac{1-\delta}{\delta}}$. In the second, we study the asymptotic behaviour of the solution.

According to the work of Varopoulos [29], the Sobolev inequality has the following consequence: let $\Omega$ be an open (not necessarily bounded) subset of $M$ and note $\Delta_{\Omega}$ the Laplacian operator with Dirichlet boundary condition. More exactly, $\Delta_{\Omega}$ is the Friedrichs extension associated to the quadratic form $u \mapsto\|d u\|_{L^{2}(\Omega)}^{2}$ defined on the closure of $C_{0}^{\infty}(\Omega)$ in $H^{1}(M)$, or, alternatively, $\Delta_{\Omega}$ is the minimal extension of $\Delta: C_{0}^{\infty}(\Omega) \longrightarrow C_{0}^{\infty}(\Omega)$. Then the heat operator $e^{-t \Delta_{\Omega}}$ associated to $\Delta_{\Omega}$ has the following mapping properties: for $p \geqslant 1$, it maps $L^{p}(\Omega)$ in $L^{\infty}(\Omega)$ and

$$
\left\|e^{-t \Delta_{\Omega}}\right\|_{L^{p} \rightarrow L^{\infty}} \leqslant C(n, p) \mu^{-n / 2 p} t^{-n / 2 p} .
$$


Moreover the operator

$$
\Delta_{\Omega}^{-\alpha / 2}=\int_{0}^{\infty} e^{-t \Delta_{\Omega}} t^{\alpha / 2-1} \frac{d t}{\Gamma(\alpha / 2)}
$$

has the following properties: if $p \in] 1, n / \alpha\left[\right.$ then it maps $L^{p}(\Omega)$ in $L^{\frac{n p}{n-p \alpha}}(\Omega)$, and

$$
\left\|\Delta_{\Omega}^{-\alpha / 2}\right\|_{L^{p} \rightarrow L^{\frac{n p}{n-p \alpha}}} \leqslant C(n, p, \alpha) \mu^{-\alpha / 2} .
$$

These properties (3.3) rely upon the bound (3.2) and a maximal theorem of E. Stein. We now want to solve the equation

$$
\Delta u+c_{n} \operatorname{Scal}_{g} u=-c_{n} \mathrm{Scal}_{g}, \quad \text { outside } K .
$$

For this we shall solve the equation

$$
\begin{aligned}
u & =\Delta_{M-K}^{-1 / 2} f \\
f+A f & =-c_{n} \Delta_{M-K}^{-1 / 2} \mathrm{Scal}_{g}
\end{aligned}
$$

where $A$ is the operator

$$
A f=\Delta_{M-K}^{-1 / 2}\left(\operatorname{Scal}_{g} \Delta_{M-K}^{-1 / 2} f\right) .
$$

The mapping properties (3.3) of the operator $\Delta_{M-K}^{-1 / 2}$ and the Hölder inequality imply that, for $1<p<n$, the operator $A$ is a bounded operator on $L^{p}(M-K)$ and

$$
\|A\|_{L^{p} \rightarrow L^{p}} \leqslant C(n, p) \mu^{-1}\left\|\operatorname{Scal}_{g}\right\|_{L^{\frac{n}{2}}(M-K)} .
$$

Moreover the hypothesis on the scalar curvature yields that $\Delta_{M-K}^{-1 / 2} \mathrm{Scal}_{g}$ lives in $L^{n \frac{1-\delta}{1+\delta}}$

$$
\left\|\Delta_{M-K}^{-1 / 2} \operatorname{Scal}_{g}\right\|_{L^{n \frac{1-\delta}{1+\delta}}(M-K)} \leqslant C(n, \delta) \mu^{-1 / 2}\left\|\operatorname{Scal}_{g}\right\|_{L^{\frac{n}{2}(1-\delta)}(M-K)} .
$$

If the compact $K$ is such that $\left(\int_{M-K} \operatorname{Scal}_{g}^{n / 2}(x) d x\right)^{\frac{2}{n}}$ is small enough, we have

$$
\|A\|_{L^{n \frac{1-\delta}{1+\delta}} \rightarrow L^{n \frac{1-\delta}{1+\delta}}} \leqslant 1 / 2
$$

and the operator $\operatorname{Id}+A$ is invertible on $L^{n \frac{1-\delta}{1+\delta}}(M-K)$. Hence we may find $f$ in $L^{n \frac{1-\delta}{1+\delta}}(M-K)$ such that

$$
f+A f=-c_{n} \Delta_{M-K}^{-1 / 2} \mathrm{Scal}_{g}
$$

The function $u=\Delta_{M-K}^{-1 / 2} f$ solves the equation (3.1) and we have

$$
\|u\|_{L^{\frac{n}{2}} \frac{1-\delta}{\delta}} \leqslant C(n, \delta) \mu^{-1}\left\|\operatorname{Scal}_{g}\right\|_{L^{\frac{n}{2}(1-\delta)}(M-K)} .
$$

In the second step of the proof, we are able to show that provided $K$ is large enough then the function $u$ is bounded by $1 / 2$ on $M-K$. For this we could employ 
the Moser iteration scheme, but we will alternatively use the mapping properties (3.3) of the previous operators.

First we show that the equation

$$
f+A f=-c_{n} \Delta_{M-K}^{-1 / 2} \mathrm{Scal}_{g}
$$

can be solved on $L^{n \frac{1-\delta}{1+\delta}} \cap L^{n \frac{1+\delta}{1-\delta}}$. First note that because of our assumptions on the scalar curvature and of (3.3), we have also

$$
\left\|\Delta_{M-K}^{-1 / 2} \operatorname{Scal}_{g}\right\|_{L^{n \frac{1+\delta}{1-\delta}}(M-K)} \leq C(n, \delta) \mu^{-1 / 2}\left\|\operatorname{Scal}_{g}\right\|_{L^{\frac{n}{2}(1+\delta)}(M-K)} .
$$

We have to show that the operator Id $+A$ is invertible on $L^{n \frac{1-\delta}{1+\delta}} \cap L^{n \frac{1+\delta}{1-\delta}}$ if $M-K$ is small enough. This stems out from a Gagliardo-Nirenberg inequality: we claim that if $h \in L^{\frac{n}{2} \frac{1-\delta}{\delta}}(M-K)$ and if $\Delta_{M-K}^{1 / 2} h \in L^{n \frac{1+\delta}{1-\delta}}$, then there is a constant $C(n, \delta, \mu)$ such that

$$
\|h\|_{L^{\infty}} \leqslant C(n, \delta, \mu)\left[\|h\|_{L^{\frac{n}{2} \frac{1-\delta}{\delta}}(M-K)}+\left\|\Delta_{M-K}^{1 / 2} h\right\|_{L^{n \frac{1+\delta}{1-\delta}}(M-K)}\right] .
$$

The Gagliardo-Nirenberg inequality is shown by mimicking the argument of [12]: we know from the subordination identity that

$$
e^{-y \sqrt{\Delta_{M-K}}}=\frac{y}{2 \sqrt{\pi}} \int_{0}^{\infty} e^{-y^{2} / 4 t} e^{-t \Delta_{M-K}} t^{-3 / 2} d t
$$

so that the Poisson operator $e^{-y \sqrt{\Delta_{M-K}}}$ maps $L^{p}$ in $L^{\infty}$ and

$$
\left\|e^{-y \sqrt{\Delta_{M-K}}}\right\|_{L^{p} \rightarrow L^{\infty}} \leqslant C(n, p) \mu^{-n / 2 p} y^{-n / p}, \forall y>0 .
$$

Now if $h$ is such a function, we have

$$
h=e^{-\sqrt{\Delta_{M-K}}} h-\int_{0}^{1} e^{-y \sqrt{\Delta_{M-K}}} \Delta_{M-K}^{1 / 2} h d y,
$$

so that we may bound $\|h\|_{L^{\infty}(M-K)}$ from above by

$$
C(n, \delta)\left[\mu^{-2 \delta /(1-\delta)}\|h\|_{L^{\frac{n}{2} \frac{1-\delta}{\delta}}(M-K)}+\mu^{(1-\delta) /(1+\delta)}\left\|\Delta_{M-K}^{1 / 2} h\right\|_{L^{n \frac{1+\delta}{1-\delta}}(M-K)}\right]
$$

which is precisely the desired inequality.

Now we already know that if $f$ lives in $L^{n \frac{1-\delta}{1+\delta}} \cap L^{n \frac{1+\delta}{1-\delta}}$ then

$$
\|A f\|_{L^{n \frac{1-\delta}{1+\delta}}} \leqslant C(n, \delta) \mu^{-1}\left\|\operatorname{Scal}_{g}\right\|_{L^{n / 2}(M-K)}\|f\|_{L^{n \frac{1-\delta}{1+\delta}}} .
$$

Moreover, (3.3) implies

$$
\|A f\|_{L^{n \frac{1+\delta}{1-\delta}(M-K)}} \leqslant C(n, \delta)\left\|\operatorname{Scal}_{g} \Delta_{M-K}^{-1 / 2} f\right\|_{L^{\frac{n}{2}(1+\delta)}(M-K)} .
$$

Now we have

$$
\left\|\operatorname{Scal}_{g} \Delta_{M-K}^{-1 / 2} f\right\|_{L^{\frac{n}{2}(1+\delta)}(M-K)} \leqslant\left\|\operatorname{Scal}_{g}\right\|_{L^{\frac{n}{2}(1+\delta)}(M-K)}\left\|\Delta_{M-K}^{-1 / 2} f\right\|_{L^{\infty}(M-K)}
$$


and the Gagliardo-Nirenberg inequality (3.5) provides

$$
\left\|\Delta_{M-K}^{-1 / 2} f\right\|_{L^{\infty}(M-K)} \leqslant C(n, \delta, \mu)\left[\left\|\Delta_{M-K}^{-1 / 2} f\right\|_{L^{\frac{n}{2} \frac{1-\delta}{\delta}}(M-K)}+\|f\|_{L^{n \frac{1+\delta}{1-\delta}(M-K)}}\right] .
$$

But again by (3.3), we have

$$
\left\|\Delta_{M-K}^{-1 / 2} f\right\|_{L^{\frac{n}{2} \frac{1-\delta}{\delta}}} \leqslant C(n, \delta) \mu^{-1 / 2}\|f\|_{L^{n \frac{1-\delta}{1+\delta}}(M-K)} .
$$

So that we end with the bound

$$
\|A f\|_{L^{n \frac{1+\delta}{1-\delta}}} \leqslant C(n, \delta, \mu)\left\|\operatorname{Scal}_{g}\right\|_{L^{\frac{n}{2}(1+\delta)}(M-K)}\left[\|f\|_{L^{n \frac{1-\delta}{1+\delta}}(M-K)}+\|f\|_{L^{\frac{n}{2} \frac{1-\delta}{\delta}}(M-K)}\right] .
$$

Hence, if $K$ is such that $\left\|\operatorname{Scal}_{g}\right\|_{L^{\frac{n}{2}(1+\delta)}(M-K)}$ and $\left\|\operatorname{Scal}_{g}\right\|_{L^{\frac{n}{2}(1-\delta)}(M-K)}$ are small enough then the operator $A$ is bounded on $L^{n \frac{1-\delta}{1+\delta}}(M-K) \cap L^{n \frac{1+\delta}{1-\delta}}(M-K)$ with an operator norm bounded by $1 / 2$.

We may then find $f \in L^{n \frac{1-\delta}{1+\delta}} \cap L^{n \frac{1+\delta}{1-\delta}}$ which solves the equation

$$
f+A f=-c_{n} \Delta_{M-K} \mathrm{Scal}_{g} \text {, outside } K \text {. }
$$

Now the function $u=\Delta_{M-K}^{-1 / 2} f$ solves the equation (3.1), and lives in $L^{\frac{n}{2} \frac{1-\delta}{\delta}}$. Moreover, $\Delta_{M-K}^{1 / 2} u=f \in L^{n \frac{1+\delta}{1-\delta}}$. We then have the bounds

$$
\begin{gathered}
\|u\|_{L^{\frac{n}{2} \frac{1-\delta}{\delta}}} \leqslant C(n, \delta, \mu)\left\|\operatorname{Scal}_{g}\right\|_{L^{\frac{n}{2}(1-\delta)}(M-K)}, \\
\|f\|_{L^{n \frac{1+\delta}{1-\delta}}} \leqslant C(n, \delta, \mu)\left\|\operatorname{Scal}_{g}\right\|_{L^{\frac{n}{2}(1+\delta)}(M-K)} .
\end{gathered}
$$

Using the Gagliardo-Nirenberg inequality (3.5), we obtain

$$
\|u\|_{L^{\infty}(M-K)} \leqslant C(n, \delta, \mu)\left[\left\|\operatorname{Scal}_{g}\right\|_{L^{\frac{n}{2}(1+\delta)}(M-K)}+\left\|\operatorname{Scal}_{g}\right\|_{L^{\frac{n}{2}(1-\delta)}(M-K)}\right] .
$$

We may then choose the compact $K$ adequately to have the desired bound $|u| \leqslant$ $1 / 2$ over $M-K$.

Remark 3.2. The Moser iteration scheme [14] would show that the function $\rho$ in the statement of Theorem 3.1 has limit zero at infinity.

Remark 3.3. If the two integrals for the scalar curvature are small with respect to the Sobolev constant, we may get a metric with vanishing scalar curvature everywhere.

Remark 3.4. It is possible to replace the hypothesis on the Sobolev inequality by a more conformally invariant one: we can assume for instance that the Yamabe invariant of a neighbourhood of infinity is positive. As already noticed, this together with boundedness of the $L^{n / 2}$-norm of the scalar curvature implies the Sobolev inequality. 


\section{On the geometry of compact manifolds with a finite number of points removed}

As already explained in the introduction, the goal of this short section is to give a proof of the following statement: if $(M, g)$ is a complete manifold controlled volume growth and finite $L^{n / 2}$-norm of Ricci curvature (1.1), the scalar curvature assumption of Theorem 3.1 together with the conclusions of Huber's theorem, then it is conformally quasi-isometric to an asymptotically locally euclidean manifold.

In fact, we shall prove the following, slightly stronger, result, which also gives some information on the case treated in section 2 .

Theorem 4.1. Let $\left(M, g_{0}\right)$ be a compact Riemanniann manifold and let $\Omega$ be a domain in $M$ endowed with a complete Riemannian metric $g$ which is conformal to $g_{0}$. Assume moreover that the Ricci and scalar curvature of $(\Omega, g)$ satisfy

$$
\operatorname{Ric}_{g} \in L^{\frac{n}{2}}, \quad \operatorname{Scal}_{g} \in L^{\frac{n}{2}(1-\delta)} \cap L^{\frac{n}{2}(1+\delta)}
$$

for some $\delta$ in $] 0,1\left[\right.$, and that $\operatorname{vol}_{g} B\left(x_{0}, r\right) \leqslant C r^{n}$ for some $x_{0}$ in $M$. Then there is a bounded smooth function $f$ on $\Omega$ such that $\left(\Omega, e^{2 f} g\right)$ has a finite number of ends, each of them asymptotically euclidean.

We recall that an end $E$ of a complete Riemannian manifold $\left(M^{n}, g\right)$ is said to be asymptotically euclidean (of order $\tau>0$ ) if $E$ is diffeomorphic to the complement of a euclidean ball in the euclidean space $\mathbb{R}^{n}$ and if, in theses coordinates, the metric $g$ satisfies

$$
\begin{array}{r}
g_{i j}(z)=\delta_{i j}+\mathrm{O}\left(|z|^{-\tau}\right), \quad \partial_{k} g_{i j}(z)=\mathrm{O}\left(|z|^{-\tau-1}\right), \\
\left|\partial_{k} g_{i j}(z)-\partial_{k} g_{i j}(w)\right||z-w|^{\alpha} \leqslant \mathrm{O}\left(\min \{|z|,|w|\}^{-\tau-1-\alpha}\right) .
\end{array}
$$

for some $\alpha \in] 0,1[$. An end of a Riemannian manifold is called asymptotically locally euclidean, or ALE in short, if a finite Riemannian cover of this end is asymptotically euclidean.

Proof. According to Theorem 2.1, we know that there is a finite set $\left\{p_{1}, \ldots, p_{k}\right\} \subset$ $M$ such that $\Omega=M-\left\{p_{1}, \ldots, p_{k}\right\}$. Theorem 3.1 provides a smooth bounded function $f$ on $\Omega$ such that the new metric $e^{2 f} g$ has vanishing scalar curvature in some neighbourhood of $\left\{p_{1}, \ldots, p_{k}\right\}$. We may then take $r>0$ small enough in order that

(i) the $g_{0}$-geodesic balls $B\left(p_{i}, r\right)$ are smooth, convex and disjoint;

(ii) the scalar curvature of $e^{2 f} g$ is zero in $B\left(p_{i}, r\right)-\left\{p_{i}\right\}$.

Fix some $i \in\{1, \ldots, k\}$. We also have existence of a bounded smooth function $h$ on $B\left(p_{i}, r\right)$ such that the metric $e^{2 h} g_{0}$ has vanishing scalar curvature. Since $g$ is conformal to $g_{0}$, there is a smooth positive function $v$ on $\Omega$ with $g=v^{\frac{4}{n-2}} g_{0}$. Now we have $e^{2 f} g=w^{\frac{4}{n-2}} e^{2 h} g_{0}$ with $w=e^{\frac{n-2}{8} f} v e^{-\frac{n-2}{8} h}$. The function $w$ is positive and satisfies

$$
\Delta w=0 \text { on } B\left(p_{i}, r\right)-\left\{p_{i}\right\}
$$


where $\Delta$ is the Laplacian associated with the metric $e^{2 h} g_{0}$. Define $\bar{w}$ to be the solution of the Dirichlet problem

$$
\begin{cases}\Delta \bar{w}=0 & \text { on } B\left(p_{i}, r\right), \\ \bar{w}=w & \text { on } \partial B\left(p_{i}, r\right) .\end{cases}
$$

The function $w-\bar{w}$ is a harmonic function on $B\left(p_{i}, r\right)-\left\{p_{i}\right\}$ and according to the maximum principle, it is a positive function. Thus, $w-\bar{w}$ is a multiple of the Green function associated to $\Delta$ on $B\left(p_{i}, r\right)$ for the Dirichlet boundary condition with pole at $p_{i}$. If we come back to $v$, we obtain that there is a constant $\lambda>0$ such that $e^{\frac{n-2}{8} f} v-\lambda G_{i}$ is a smooth function in $B\left(p_{i}, r\right)$ where $G_{i}$ is the conformal Green function of $\left(B\left(p_{i}, r\right), g_{0}\right)$ with Dirichlet boundary condition and pole at $p_{i}$. Computations done by J. Lee and T. Parker [22, Lemma 6.4] show that the new metric $e^{2 f} g$ is asymptotically euclidean.

Keeping the terminology of [22], we shall say that $\Omega$ is obtained from $\left(M, g_{0}\right)$ by a stereographic conformal blow up.

\section{Structure at infinity of conformally flat manifolds}

This section is the central part of the paper. We begin in the first subsection by proving that scalar flat, conformally flat complete manifolds satisfying both condition (1.1) on Ricci curvature and the Sobolev inequality

$$
\mu_{n}(M, g)\left(\int_{M} u^{\frac{2 n}{n-2}}\right)^{1-2 / n} \leqslant \int_{M}|d u|^{2}, \quad \forall u \in C_{0}^{\infty}(M)
$$

are asymptotically locally euclidean. This and the uniformization arguments of the previous sections are immediatly used in the following subsection, thus proving our main result, Theorem 5.7, in dimension 4 . The last subsection deals with the case of other dimensions.

Geometry at infinity of scalar flat, conformally flat manifolds. Our second main technical result is the following:

Theorem 5.1. Let $\left(M^{n}, g\right)$ be a Riemannian manifold which satisfies the Sobolev inequality

$$
\mu_{n}(M, g)\left(\int_{M} u^{\frac{2 n}{n-2}}\right)^{1-2 / n} \leqslant \int_{M}|d u|^{2}, \quad \forall u \in C_{0}^{\infty}(M),
$$

whose Ricci tensor satisfies

$$
\int_{M}\left|\operatorname{Ric}_{g}\right|^{\frac{n}{2}}(x) d \operatorname{vol}_{g}(x)<\infty .
$$

If $(M, g)$ is scalar flat and conformally flat in a neighbourhood of infinity, then it has a finite number of ends, each of them asymptotically locally euclidean. 
Proof. The idea is to show that the tracefree Ricci tensor Ric ${ }_{0}$ decays at infinity faster than expected. Fix a point $p$ in $M$. If we are able to prove that

$$
\left|\operatorname{Ric}_{0}\right|(x) \leqslant \frac{C}{d(p, x)^{2+\eta}} \quad \forall x \in M-B_{p}(1),
$$

for some $\eta>0$, then we can apply the characterization of asymptotically locally euclidean manifolds due to A. Kasue, S. Bando and H. Nakajima [4]: the manifold is indeed asymptotically locally euclidean if curvature has the decay (5.1) above and if the volume of $B_{p}(r)$ grows at least as $r^{n}$. The first assumption is then obviously satisfied (as the metric is scalar flat and conformally flat), whereas the second is a well-known consequence of the Sobolev inequality [7, Proposition 2.4].

We now have to exhibit the estimate (5.1). Our starting point is the basic Weitzenböck formula for Ricci curvature [5, Formula (4.1)]

$$
\left(d^{D} \delta^{D}+\delta^{D} d^{D}\right) \operatorname{Ric}_{0}=D^{*} D \operatorname{Ric}_{0}+c \operatorname{Ric}_{0} \operatorname{Ric}_{0},
$$

where $c$ is a constant whose value is irrelevant for our concerns and may then vary from line to line. We may apply this to our conformally flat, scalar flat, metric outside a compact set. The Ricci tensor is then a closed and co-closed 1-form with values into 1 -forms and we get

$$
\Delta \operatorname{Ric}_{0}=D^{*} D \operatorname{Ric}_{0}=c \operatorname{Ric}_{0} \circ \operatorname{Ric}_{0} .
$$

We can immediately infer from this a first subelliptic estimate for the norm $\mid$ Ric $_{0} \mid$ :

$$
\begin{aligned}
\frac{1}{2} \Delta\left|\operatorname{Ric}_{0}\right|^{2} & =\left|\operatorname{Ric}_{0}\right| \Delta\left|\operatorname{Ric}_{0}\right|-|d| \text { Ric }\left._{0}\right|^{2} \\
& =\left\langle\operatorname{Ric}_{0}, \Delta \operatorname{Ric}_{0}\right\rangle-\left|D \operatorname{Ric}_{0}\right|^{2}
\end{aligned}
$$

Hence, by Kato inequality,

$$
\left|\operatorname{Ric}_{0}\right| \Delta\left|\operatorname{Ric}_{0}\right|=\left\langle\operatorname{Ric}_{0}, \Delta \operatorname{Ric}_{0}\right\rangle-\left|D \operatorname{Ric}_{0}\right|^{2}+\left.|d| \operatorname{Ric}_{0}\right|^{2} \leqslant c\left|\operatorname{Ric}_{0}\right|^{2},
$$

and finally

$$
\Delta\left|\operatorname{Ric}_{0}\right| \leqslant c\left|\operatorname{Ric}_{0}\right|^{2},
$$

in the weak sense, outside a compact set.

The next ingredient is the refined Kato-like inequality proven in [6]; for $\mathrm{Ric}_{0}$ is in the kernel of the elliptic first order operator $d^{D}+\delta^{D}$ acting on trace-free symmetric tensors, we have

$$
|d| \operatorname{Ric}_{0}|| \leqslant \sqrt{\frac{n}{n+2}}\left|D \operatorname{Ric}_{0}\right|
$$

wherever $\operatorname{Ric}_{0}$ does not vanish (see $[6]$ for the precise computation). Letting $\beta=$ 
$\frac{n-2}{n}$, we may now compute:

$$
\begin{aligned}
\Delta\left(\left|\operatorname{Ric}_{0}\right|^{\beta}\right)= & \beta\left(\left|\operatorname{Ric}_{0}\right|^{\beta-1} \Delta\left|\operatorname{Ric}_{0}\right|-\left.(\beta-1)\left|\operatorname{Ric}_{0}\right|^{\beta-2}|d| \operatorname{Ric}_{0}\right|^{2}\right) \\
= & \beta\left|\operatorname{Ric}_{0}\right|^{\beta-2}\left(\left\langle\operatorname{Ric}_{0}, \Delta \operatorname{Ric}_{0}\right\rangle-\left|D \operatorname{Ric}_{0}\right|^{2}+\left.|d| \operatorname{Ric}_{0}\right|^{2}\right) \\
& \quad-\beta(\beta-1)\left|\operatorname{Ric}_{0}\right|^{\beta-2}|d| \operatorname{Ric}_{0}||^{2} \\
= & \beta\left|\operatorname{Ric}_{0}\right|^{\beta-2}\left(\left\langle\operatorname{Ric}_{0}, \Delta \operatorname{Ric}_{0}\right\rangle-\left|D \operatorname{Ric}_{0}\right|^{2}+\left.(2-\beta)|d| \operatorname{Ric}_{0}\right|^{2}\right) .
\end{aligned}
$$

Taking now into account the precise value of $\beta$, we end up with

$$
\Delta\left(\left|\operatorname{Ric}_{0}\right|^{\frac{n-2}{n}}\right) \leqslant \frac{n-2}{n}\left|\operatorname{Ric}_{0}\right|^{-\frac{n+2}{n}}\left|\left\langle\operatorname{Ric}_{0}, \Delta \operatorname{Ric}_{0}\right\rangle\right| \leqslant C\left|\operatorname{Ric}_{0}\right|^{\frac{2(n-1)}{n}}
$$

in the weak sense outside a compact set.

Denoting $u=\left|\operatorname{Ric}_{0}\right|$ and $v=\left|\operatorname{Ric}_{0}\right|^{1 / \gamma}$ where $\gamma=\frac{n}{n-2}$, our general assumptions imply that $u$ is in $L^{n / 2}$, and that

$$
\Delta u \leqslant c u^{2} \text {, and } \Delta v \leqslant c u v \text { outside a compact set. }
$$

The conclusion will now follow from the next two lemmas.

In what follows, we denote by $M_{r}$ the complement of the ball of radius $r$ and centre $p$ in $M$.

Lemma 5.2. Assume that $M$ satisfies the Sobolev inequality. Let $u$ positive be in $L^{n / 2}$ such that $\Delta u \leqslant c u^{2}$ in the weak sense. Then there exists $r_{0}$ such that for all $r \geqslant r_{0}, u$ belongs to $L^{\infty}\left(M_{r}\right)$ and

$$
\sup _{M_{2 r}} u \leqslant C r^{-2}\left(\int_{M_{r}} u^{\frac{n}{2}}\right)^{\frac{2}{n}} \text {. }
$$

for $r$ large enough.

Lemma 5.3. Assume that $M$ satisfies the Sobolev inequality and has sub-euclidean volume growth. Let $u$ positive be in $L^{n / 2}$, $v$ positive in $L^{\gamma n / 2}$ with $\gamma=n /(n-2)$. Assume $\Delta v \leqslant$ cuv in the weak sense, then

$$
\left(\int_{M_{2 r}} v^{\gamma \frac{n}{2}}\right)^{\frac{1}{\gamma}} \leqslant\left(\int_{M_{r}-M_{2 r}} v^{\gamma \frac{n}{2}}\right)^{\frac{1}{\gamma}}
$$

for $r$ large enough.

Proof of Theorem 5.1 (assuming the lemmas). We begin by applying Lemma 5.2. Since $u=\left|\operatorname{Ric}_{0}\right|$ belongs to $L^{n / 2}$,

$$
\sup _{M_{2 r}} u \leqslant C r^{-2}\left(\int_{M_{r}} u^{\frac{n}{2}}\right)^{\frac{2}{n}}=o\left(r^{-2}\right) .
$$

The second step is to apply Lemma 5.3 and we need volume growth control from above. Now remember Scal and $W$ vanish, whereas $u$ above is the norm of the 
tracefree Ricci tensor. Hence $K_{g}=o\left(r^{-2}\right)$ and diameter is controlled from above and volume growth is controlled from below (from the Sobolev inequality) on each annulus $M_{2 k r}-M_{k^{-1} r}$. We can then infer from Anderson-Cheeger harmonic radius' theory $[2,3,16,23]$ that the rescaled annuli $\left(M_{k r_{i}}-M_{k^{-1} r_{i}}, r_{i}^{-2} g\right)$ are covered by a finite (and uniformly bounded) number of balls of uniformly bounded size where the metric coefficients are $C^{1, \alpha}$-close to the euclidean metric. Hence one gets volume growth control from above. We can now estimate further the tracefree part of Ricci by applying Lemma 5.3 to $v=u^{1 / \gamma}$. We get

$$
\left(\int_{M_{2 r}} v^{\gamma \frac{n}{2}}\right)^{\frac{1}{\gamma}} \leqslant\left(\int_{M_{r}-M_{2 r}} v^{\gamma \frac{n}{2}}\right)^{\frac{1}{\gamma}}
$$

hence

$$
\int_{M_{2 r}} u^{\frac{n}{2}} \leqslant \int_{M_{r}} u^{\frac{n}{2}}-\int_{M_{2 r}} u^{\frac{n}{2}}
$$

The conclusion now stems out from injecting the result of the following elementary Lemma into estimate (5.10) above.

Lemma 5.4. If $F$ is a positive non-increasing function satisfying, for $r \geqslant 1$,

$$
F(2 r) \leqslant C(F(r)-F(2 r)) \quad \text { and } \quad \lim _{r \rightarrow \infty} F(r)=0
$$

then there exists $\eta>0$ such that $F(r) \leqslant C r^{-\eta}$ for large $r$.

We now detail the proofs of the first two Lemmas, leaving the third one to the reader.

Proof of Lemma 5.2. We shall use the standard Moser iteration scheme. It simplifies matters to take $r=1$, so that we will prove that, if $w$ is a positive function in $L^{n / 2}$ satisfies $\Delta w \leqslant c w^{2}$ in the weak sense, then there is $\epsilon_{0}>0$ such that, if

$$
\left(\int_{M_{1}} w^{\frac{n}{2}}\right)^{\frac{2}{n}}<\varepsilon_{0}
$$

then

$$
\sup _{M_{2}} w \leqslant C\left(\int_{M_{1}} w^{\frac{n}{2}}\right)^{\frac{2}{n}}
$$

Rescaling by $g \rightarrow r^{-2} g$ where $r \geqslant r_{0}$ and $r_{0}$ is chosen such that $\|u\|_{L^{n / 2}\left(M_{r_{0}}\right)}<\varepsilon_{0}$ establishes the link with the desired property.

Let $1<r_{-}<r_{+}<<r^{\prime}<\infty$ and let $\varphi$ be a smooth cut-off function with support in $M_{r_{-}}-M_{2 r^{\prime}}$ having value 1 in $M_{r_{+}}-M_{r^{\prime}}$ and such that $|d \varphi| \leqslant$ 
$1 /\left(r_{+}-r_{-}\right)$. Let moreover $\alpha \geqslant 1$. We now compute:

$$
\begin{aligned}
\frac{4 \alpha}{(\alpha+1)^{2}} \int \varphi^{2}\left|d\left(w^{\frac{\alpha+1}{2}}\right)\right|^{2} & =\int \varphi^{2}\left\langle d\left(w^{\alpha}\right), d w\right\rangle \\
& =\int \varphi^{2} w^{\alpha} \Delta w-2 \int \varphi w^{\alpha}\langle d \varphi, d w\rangle \\
& \leqslant c \int \varphi^{2} w^{\alpha+2}+2 \int \varphi w^{\alpha}|d \varphi||d w|,
\end{aligned}
$$

where the last line has been obtained with the inequation (5.8). By Young's inequality

$$
\left(2 w^{\frac{\alpha+1}{2}}|d \varphi|-\frac{1}{4} \varphi w^{\frac{\alpha-1}{2}}|d w|\right)^{2} \geqslant 0
$$

i.e.

$$
4 w^{\alpha+1}|d \varphi|^{2}+\frac{1}{4(\alpha+1)^{2}} \varphi^{2}\left|d\left(w^{\frac{\alpha+1}{2}}\right)\right|^{2} \geqslant \varphi w^{\alpha}|d \varphi||d w|,
$$

applied to the last term, we get

$$
\frac{4\left(\alpha-\frac{1}{8}\right)}{(\alpha+1)^{2}} \int \varphi^{2}\left|d\left(w^{\frac{\alpha+1}{2}}\right)\right|^{2} \leqslant c \int \varphi^{2} w^{\alpha+2}+8 \int w^{\alpha+1}|d \varphi|^{2}
$$

which is easily turned into

$$
\int\left|d\left(\varphi w^{\frac{\alpha+1}{2}}\right)\right|^{2} \leqslant c \alpha\left(\int \varphi^{2} w^{\alpha+2}+\int w^{\alpha+1}|d \varphi|^{2}\right) .
$$

We may now conclude from the Sobolev inequality that (recall $\gamma=\frac{n}{n-2}$ )

$$
\left(\int \varphi^{2 \gamma} w^{\gamma(\alpha+1)}\right)^{\frac{1}{\gamma}} \leqslant c \alpha\left(\int \varphi^{2} w^{\alpha+2}+\int w^{\alpha+1}|d \varphi|^{2}\right) .
$$

Step 1. Suppose now $\alpha+1=\frac{n}{2}$. The formula (5.11) above yields

$$
\begin{aligned}
\left(\int \varphi^{2 \gamma} w^{\gamma \frac{n}{2}}\right)^{\frac{1}{\gamma}} & \leqslant c(n)\left(\int \varphi^{2} w^{\frac{n}{2}+1}+\int w^{\frac{n}{2}}|d \varphi|^{2}\right) \\
& \leqslant c(n)\left(\left(\int \varphi^{2 \gamma} w^{\frac{n}{2} \gamma}\right)^{\frac{1}{\gamma}}\left(\int_{\operatorname{supp} \varphi} w^{\frac{n}{2}}\right)^{\frac{2}{n}}+\int w^{\frac{n}{2}}|d \varphi|^{2}\right) .
\end{aligned}
$$

Hence if $\|w\|_{L^{\frac{n}{2}}\left(M_{1}\right)}$ is small enough, we can absorb this term in the left hand side and conclude that

$$
\left(\int \varphi^{2 \gamma} w^{\gamma \frac{n}{2}}\right)^{\frac{1}{\gamma}} \leqslant c(n) \int|d \varphi|^{2} w^{\frac{n}{2}}
$$

so that $\|w\|_{\gamma \frac{n}{2}} \leqslant C\|w\|_{\frac{n}{2}}$ by letting $r^{\prime}$ tend to infinity (and $C$ is a constant strongly depending on the geometry of $M$ ). 
Step 2. Consider now the general case $\alpha+1>\frac{n}{2}$. Fix $q$ in $] n, \gamma n[$, we may estimate

$$
\int \varphi^{2} w^{\alpha+2} \leqslant\left(\int_{\operatorname{supp} \varphi} w^{\frac{q}{2}}\right)^{\frac{2}{q}}\left(\int \varphi^{\frac{2 q}{q-2}} w^{\frac{\alpha+1}{2} \frac{2 q}{q-2}}\right)^{\frac{q-2}{q}} .
$$

Standard Hölder-like interpolation inequalities enable us to control the first parenthesis on the right-hand side by the $L^{\frac{n}{2}}$ and $L^{\gamma \frac{n}{2}}$ norms of $w$. The second parenthesis is estimated as follows:

$$
\left(\int \varphi^{\frac{2 q}{q-2}} w^{\frac{\alpha+1}{2} \frac{2 q}{q-2}}\right)^{\frac{q-2}{q}} \leqslant \varepsilon\left(\int \varphi^{\frac{2 n}{n-2}} w^{\frac{\alpha+1}{2} \frac{2 n}{n-2}}\right)^{\frac{n-2}{n}}+\varepsilon^{-\nu} \int \varphi^{2} w^{\alpha+1}
$$

with $\nu^{-1}=\frac{q}{n}-1$ and $0<\varepsilon<1$ to be chosen later. The basic inequality (5.11) then becomes

$$
\left(\int\left(\varphi w^{\frac{\alpha+1}{2}}\right)^{2 \gamma}\right)^{\frac{1}{\gamma}} \leqslant c \alpha\left(\varepsilon\left\|\varphi w^{\frac{\alpha+1}{2}}\right\|_{2 \gamma}^{2}+\varepsilon^{-\nu}\left\|\varphi w^{\frac{\alpha+1}{2}}\right\|_{2}^{2}\right)+c \alpha \int w^{\alpha+1}|d \varphi|^{2} .
$$

For a given $\alpha$, we may now choose $\varepsilon=\frac{1}{2}(c \alpha)^{-1}$ and absorb the first term on the right-hand side in the left-hand side and obtain the second basic estimate:

$$
\left\|\varphi w^{\frac{\alpha+1}{2}}\right\|_{2 \gamma}^{2} \leqslant c \alpha\left(1+\alpha^{\nu}\right)\left\|(\varphi+|d \varphi|) w^{\frac{\alpha+1}{2}}\right\|_{2}^{2}
$$

Let now $p=\alpha+1$ and

$$
N(p, r)=\left(\int_{M_{r}} w^{p}\right)^{\frac{1}{p}}
$$

By letting $r^{\prime}$ go to infinity, we may reinterpret formula (5.12) as

$$
N\left(\gamma p, r_{+}\right) \leqslant\left(\frac{c p\left(1+p^{\nu}\right)}{r_{+}-r_{-}}\right)^{\frac{2}{p}} N\left(p, r_{-}\right) .
$$

This is easily iterated, letting $p_{0}=\frac{n}{2}, p_{m}=\gamma^{m} p_{0}, r_{m,-}=1+2^{-(m+2)}$ and $r_{m,+}=2-2^{-(m+2)}$, thus leading to:

$$
N\left(\gamma^{m} p_{0}, r_{m,+}\right) \leqslant(c \gamma)^{c \sum_{i=1}^{m} i \gamma^{-i}} N\left(\gamma^{m-1} p_{0}, r_{m,-}\right) .
$$

Letting $m$ tend to infinity yields the expected result.

We now pass on to Lemma 5.3, whose proof looks quite similar.

Proof of Lemma 5.3. Arguing as in the previous Lemma, using the inequation and the Sobolev inequality, we arrive with some computations to another basic estimate involving the two functions $u$ and $v$ and an extra cut-off function $\varphi$ with support in $M_{r}$, having value 1 on $M_{2 r}$ and such that $|d \varphi| \leqslant 1 / r$ :

$$
\left(\int\left(\varphi v^{\frac{n}{4}}\right)^{\frac{2 n}{n-2}}\right)^{\frac{n-2}{n}} \leqslant c\left(\int u \varphi^{2} v^{\frac{n}{2}}+\int v^{\frac{n}{2}}|d \varphi|^{2}\right) .
$$


We may then apply the Hölder inequality to the first term in the right-hand side, and absorb it in the left-hand side if $r$ is chosen large enough. We are left with

$$
\left(\int \varphi^{2} v^{\gamma \frac{n}{2}}\right)^{\frac{1}{\gamma}} \leqslant c \int v^{\frac{n}{2}}|d \varphi|^{2}
$$

and with Hölder again,

$$
\left(\int \varphi^{2} v^{\gamma \frac{n}{2}}\right)^{\frac{1}{\gamma}} \leqslant c\left(\int_{\operatorname{supp} d \varphi} v^{\gamma \frac{n}{2}}\right)^{\frac{1}{\gamma}}\left(\int|d \varphi|^{n}\right)^{\frac{2}{n}} .
$$

We may now use our assumption on $d \varphi$ together with the bound on volume growth and conclude that

$$
\left\|v^{\gamma}\right\|_{L^{\frac{n}{2}}\left(M_{2 r}\right)} \leqslant C\left(\left\|v^{\gamma}\right\|_{L^{\frac{n}{2}}\left(M_{r}-M_{2 r}\right)}\right)
$$

which ends the proof.

Remark 5.5. The asymptotic structure is here $C^{1, \alpha}$-asymptotically flat. It may then be not regular enough for our purposes. Since we intend to apply methods of [17], we a priori need $C^{3, \alpha}$-asymptotic flatness. However, the elliptic trick of [17, Lemme 4.4] may be used to obtain more regularity in weighted Hölder spaces through a further conformal rescaling.

Geometry at infinity of 4-dimensional conformally flat manifolds. We prove here our main result: any complete conformally flat 4-dimensional manifold $(M, g)$ satisfying

$$
\operatorname{Ric}_{g} \in L^{2}, \quad \operatorname{Scal}_{g} \in L^{\frac{4}{3}} \cap L^{2(1+\delta)}, \quad \text { with } \delta>0
$$

is conformally equivalent to a compact manifold with a finite number of points removed. As the proof shows, it is enough to consider manifolds that are conformally flat around infinity only. We begin by stating:

Lemma 5.6. Let $(M, g)$ be a complete Riemannian manifold of dimension 4 which is conformally flat in a neighbourhood of infinity. Assume it satisfies the Sobolev inequality

$$
\mu(M, g)\left(\int_{M} u^{4}\right)^{1 / 2} \leqslant \int_{M}|d u|^{2}, \quad \forall u \in C_{0}^{\infty}(M)
$$

and that the Ricci and scalar curvatures of $(M, g)$ satisfy

$$
\left.\operatorname{Ric}_{g} \in L^{2}, \quad \operatorname{Scal}_{g} \in L^{\frac{4}{3}} \cap L^{2(1+\delta)} \text { for some } \delta \in\right] 0,1[,
$$

then there is a bounded smooth function $f$ on $M$ such that $\left(M, e^{2 f} g\right)$ has a finite number of ends, each of them asymptotically locally euclidean.

Proof. According to Theorem 3.1, there is a positive function $v=1+u$ such that 
(i) there is a positive constant $C$ with $C^{-1} \leqslant v \leqslant C$;

(ii) the new metric $v^{2} g$ has vanishing scalar curvature outside some compact set;

(iii) the function $u$ is in $L^{4} \cap L^{\infty}$ and it satisfies the equation

$$
\Delta u+\frac{1}{6} \operatorname{Scal}_{g} u=\varphi
$$

where $\varphi=-\frac{1}{6} \mathrm{Scal}_{g}$ outside some compact set.

In order to apply Theorem 5.1, we need integrability of the Ricci curvature of $v^{2} g$ to the power $n / 2=2$. The formula for the change of Ricci curvature under a conformal change of metric $\bar{g}=v^{2} g$ is

$\operatorname{Ric}_{\bar{g}}=\operatorname{Ric}_{g}-(n-2) v^{-1} D d v+2(n-2) v^{-2} d v \otimes d v+\left(v^{-1} \Delta v-(n-3) v^{-2}|d v|^{2}\right)$.

Hence, we need to show that

(i) $\quad d u \in L^{4}$ and

(ii) $D d u \in L^{2}$.

We first notice that $d u$ is in $L^{2}$ : from the proof of Theorem 3.1 (and keeping the notations thereof), we have found $u$ as a solution of the equation

$$
\begin{aligned}
u & =\Delta_{M-K}^{-1 / 2} f \\
f+A f & =-c_{n} \Delta_{M-K}^{-1 / 2} \mathrm{Scal}_{g}
\end{aligned}
$$

where $A$ is the operator

$$
f \longmapsto A f=\Delta_{M-K}^{-1 / 2}\left(\operatorname{Scal}_{g} \Delta_{M-K}^{-1 / 2} f\right) .
$$

Moreover our assumption on the scalar curvature implied that $f$ lived in $L^{2}(M-K)$. Let $H_{0}^{1}(M-K)$ be the completion of the space $C_{0}^{\infty}(M-K)$ endowed with the norm $h \mapsto\|d h\|_{L^{2}}$, then the operator $\Delta_{M-K}^{-1 / 2}$ realizes an isometry between $L^{2}(M-K)$ and $H_{0}^{1}(M-K)$. Hence $u=\Delta_{M-K}^{-1 / 2} f$ is in the Sobolev space $H_{0}^{1}(M-K)$; hence $d u$ lives in $L^{2}$.

Now we let $\alpha=d u$ and the Bochner identity implies that the 1-form $\alpha$ solves the equation

$$
\Delta \alpha+\operatorname{Ric}(\alpha)+\frac{1}{6} \operatorname{Scal}_{g} \alpha=-\frac{1}{6} u d \operatorname{Scal}_{g}+d \varphi .
$$

where $\Delta=D^{*} D$ is the rough Laplacian. From an integration by parts formula, we get

$$
\begin{aligned}
\int_{M}|D(\psi \alpha)|^{2} & =\int_{M}|d \psi|^{2} \alpha^{2}+\int_{M}\langle\Delta \alpha, \alpha\rangle \psi^{2} \\
= & \int_{M}|d \psi|^{2} \alpha^{2}-\int_{M}\left\langle\left(\operatorname{Ric}_{g}+\frac{1}{6} \operatorname{Scal}_{g}\right) \alpha, \alpha\right\rangle \psi^{2} \\
& -\frac{1}{6} \int_{M} \psi^{2} u\left\langle d \operatorname{Scal}_{g}, \alpha\right\rangle+\int_{M} \psi^{2}\langle d \varphi, \alpha\rangle
\end{aligned}
$$


Let $R>0$ and let $\psi_{R}$ be a cutoff function with value 1 in $B\left(x_{0}, R\right)$ and 0 outside $B\left(x_{0}, 2 R\right)$. We choose $\psi=\psi_{R}-\psi_{R_{0}},\left(R>R_{0}\right)$ such that $\varphi=-\frac{1}{6} \mathrm{Scal}_{g}$ outside $B\left(x_{0}, R_{0}\right)$, and we integrate by parts in order to get rid of the term in $d \mathrm{Scal}_{g}$ :

$$
\begin{aligned}
\int_{M}|D(\psi \alpha)|^{2}= & \int_{M}|d \psi|^{2} \alpha^{2}-\int_{M} \psi^{2}\left\langle\left(\operatorname{Ric}_{g}+\frac{1}{6} \operatorname{Scal}_{g}\right) \alpha, \alpha\right\rangle \\
& -\frac{1}{6} \int_{M} \psi^{2}\left\langle d \operatorname{Scal}_{g}, \alpha\right\rangle(1+u) \\
= & \int_{M}|d \psi|^{2} \alpha^{2}-\int_{M} \psi^{2}\left\langle\left(\operatorname{Ric}_{g}+\frac{1}{6} \operatorname{Scal}_{g}\right) \alpha, \alpha\right\rangle \\
& +\left(\frac{1}{6}\right)^{2} \int_{M} \psi^{2} \operatorname{Scal}_{g}^{2}(1+u)^{2}+\frac{1}{6} \int_{M} \psi^{2} \operatorname{Scal}_{g}|\alpha|^{2} \\
& +\frac{1}{3} \int_{M} \psi \operatorname{Scal}_{g}(1+u)\langle d \psi, \alpha\rangle
\end{aligned}
$$

With the Cauchy-Schwarz inequality, we get

$$
\int_{M}|D(\psi \alpha)|^{2} \leqslant 2 \int_{M}|d \psi|^{2} \alpha^{2}+\int_{M} \psi^{2}\left|\mathrm{Ric}_{-}\right||\alpha|^{2}+\frac{1}{18} \int_{M} \psi^{2} \operatorname{Scal}_{g}^{2}(1+u)^{2} .
$$

Now we use the Sobolev inequality and a Hölder inequality.

Letting $B=B\left(x_{0}, 2 R_{0}\right)$, we have:

$$
\begin{aligned}
\left(\frac{\mu}{2}-\| \text { Ric }_{-} \|_{L^{2}(M-B)}\right) & \left(\int_{M}(\psi|\alpha|)^{4}\right)^{1 / 2}+\frac{1}{2} \int_{M}|D(\psi \alpha)|^{2} \\
& \leqslant 2 \int_{M}|d \psi|^{2} \alpha^{2}+\frac{1}{18} \int_{M} \operatorname{Scal}_{g}^{2}(1+u)^{2} \psi^{2} .
\end{aligned}
$$

We can then choose $R_{0}$ such that

$$
\| \text { Ric }_{-} \|_{L^{2}\left(M-B\left(x_{0}, 2 R_{0}\right)\right)} \leqslant \mu / 4 \text {. }
$$

Since $\alpha \in L^{2}$ and Scal $_{g} \in L^{2}$ by assumption, letting $R$ go to infinity yields $\alpha \in L^{4}$ and $D \alpha \in L^{2}$.

Applying the compactification theorem for conformally flat asymptotically euclidean manifolds proved by the second author [17, Corollaire B.1] immediately yields:

Theorem 5.7. Let $(M, g)$ be a complete Riemannian manifold of dimension 4 which is conformally flat in a neighbourhood of infinity. Assume it satisfies the Sobolev inequality

$$
\mu(M, g)\left(\int_{M} u^{4}\right)^{1 / 2} \leqslant \int_{M}|d u|^{2}, \quad \forall u \in C_{0}^{\infty}(M)
$$

and the Ricci and scalar curvatures of $(M, g)$ satisfy

$$
\left.\operatorname{Ric}_{g} \in L^{2}, \operatorname{Scal}_{g} \in L^{\frac{4}{3}} \cap L^{2(1+\delta)} \text { for some } \delta \in\right] 0,1[
$$


then $(M, g)$ is conformally equivalent to a compact orbifold with a finite number of singular points removed.

Remark 5.8. Stefan Unnebrink has constructed asymptotically flat (in curvature sense) metrics on $\mathbb{R}^{4}$ with curvature bounded by $r^{-2-\varepsilon}$ (with $\varepsilon>0$ ) and slow volume growth, namely $\operatorname{vol}_{g} B\left(x_{0}, r\right) \leqslant C r^{3}[28]$. Hence, it seems necessary to have an assumption similar to the Sobolev inequality, which ensures euclidean volume growth (as is the case on any ALE manifold). It would be nice to know which assumptions imply the Sobolev inequality in the conformally flat case (apart from the already quoted positive Yamabe invariant case).

Other dimensions. Careful examination of the proof above shows that it relies on the special fact that $L^{\frac{n}{2}}=L^{2}$ has a Hilbert space structure in dimension 4 . It then breaks down for $n \neq 4$. Unfortunately, we have been unable to find an alternative argument that would cover the general case. We shall however prove here that analogous results can be obtained in dimensions $n \neq 4$ with a strengthened assumption on Ricci curvature.

Theorem 5.9. Let $(M, g)$ be a complete Riemannian manifold of dimension $n$ which is conformally flat in a neighbourhood of infinity. Assume it satisfies the Sobolev inequality

$$
\mu_{n}(M, g)\left(\int_{M} u^{\frac{2 n}{n-2}}\right)^{1-\frac{2}{n}} \leqslant \int_{M}|d u|^{2}, \quad \forall u \in C_{0}^{\infty}(M),
$$

and the Ricci and scalar curvatures of $(M, g)$ satisfy

$$
\left.r^{2}\left|\operatorname{Ric}_{g}\right| \leqslant C, \operatorname{Ric}_{g} \in L^{\frac{n}{2}} \text {, and } \operatorname{Scal}_{g} \in L^{\frac{n}{2}(1-\delta)} \text { for some } \delta \in\right] 0,1[
$$

where $r$ is the distance function to a fixed point. Then $(M, g)$ is conformally equivalent to a compact orbifold with a finite number of singular points removed.

Proof. As above, the main problem is to show that $d u$ is in $L^{n}$ and $D d u$ in $L^{n / 2}$. The idea is here to use classical local elliptic estimates for relatively compact domains in $M$ : for each $\omega^{\prime} \subset \subset \omega \subset \subset M$, there is a constant $C\left(\omega, \omega^{\prime}\right)>0$ such that

$$
\|u\|_{W^{2, \frac{n}{2}}} \leqslant C\left(\omega, \omega^{\prime}\right)\left(\left\|\Delta u+c_{n} \operatorname{Scal}_{g} u\right\|_{L^{\frac{n}{2}}}+\|u\|_{L^{\frac{n}{2}}}\right) .
$$

If one intends to stick to finiteness of $L^{n / 2}$-norm of Ricci curvature (1.1), without any extra assumption, one encounters the following obstacle: there is a priori no way to estimate uniformly (w.r.t. the domains $\omega$ and $\left.\omega^{\prime}\right)$ the constants $C\left(\omega, \omega^{\prime}\right)$, and it seems then impossible to globalize such estimates on the whole manifold. Assuming the extra condition stated in formula (5.14) is the key to overcoming the problem. Let $A_{s}$ be the geodesic annulus $A(s / 2,2 s)$ from a fixed basepoint $x_{0}$. Then the annuli $A_{s}$ with metric $g_{s}=s^{-2} g$ have uniformly bounded Ricci curvature, 
are conformally flat and satisfy the Sobolev inequality. From Cheeger's Lemma $[10,11]$ (see also $[23,10.4 .5]$ ), their injectivity radii are uniformly bounded from below. Hence they have uniform lower bound on the harmonic radius $[2,3,16]$ : $r_{H}\left(g_{s}\right) \geqslant r_{0}>0$. On each ball of radius $r_{0} / 2$, there exist harmonic coordinates and the metric coefficients are there controlled in the $C^{1, \alpha}$-topology.

This has two useful consequences: first of all it gives volume growth control, as above, and moreover one may apply the local elliptic estimate above, extracted from [14, Theorem 9.11], in euclidean norms, to $\omega=B\left(r_{0} / 2\right), \omega^{\prime}=B\left(r_{0} / 4\right)$ and the equation

$$
\Delta_{g_{s}} u+c_{n} \operatorname{Scal}_{g_{s}} u=c_{n} \varphi_{s}
$$

where $\varphi_{s}=-\operatorname{Scal}_{g_{s}}$ around infinity. Thus,

$$
\|u\|_{W^{2, \frac{n}{2}}\left(B\left(r_{0} / 4\right)\right)} \leqslant C_{0}\left(\left\|\Delta_{g_{s}} u+c_{n} \operatorname{Scal}_{g_{s}} u\right\|_{L^{\frac{n}{2}}\left(B\left(r_{0} / 2\right)\right)}+\|u\|_{L^{\frac{n}{2}}\left(B\left(r_{0} / 2\right)\right)}\right) .
$$

From the euclidean Sobolev inequality, one gets

$$
\|D d u\|_{L^{\frac{n}{2}}\left(B\left(r_{0} / 4\right)\right)}+\|d u\|_{L^{n}\left(B\left(r_{0} / 4\right)\right)} \leqslant C_{1}\left(\left\|\varphi_{s}\right\|_{L^{\frac{n}{2}}\left(B\left(r_{0} / 2\right)\right)}+\|u\|_{L^{\frac{n}{2}}\left(B\left(r_{0} / 2\right)\right)}\right)
$$

and the bounds on the metric coefficients in the coordinates yield that the same inequality is valid with $g_{s}$-dependent norms rather than coordinate-dependent ones. Coming back to $g$, using the volume bound from above obtained at the beginning of this proof and the bound from below proved in formula (2.3) which yield a uniform upper bound on the number of harmonic balls needed to cover each annulus and the conformal covariance of each norm involved, we end up with

$$
\|D d u\|_{L^{\frac{n}{2}(M)}}+\|d u\|_{L^{n}(M)} \leqslant C_{2}\left(\|\varphi\|_{L^{\frac{n}{2}(M)}}+\left\|r^{-2} u\right\|_{L^{\frac{n}{2}}(M)}\right) .
$$

Section 3 tells us that $u$ lives in $L^{\frac{n}{2} \frac{1-\delta}{\delta}}$ and the last term can then be estimated by Hölder's inequality and the volume growth. This ends the proof.

Remark 5.10. If one wants to keep only integral conditions, one can obtain the same conclusions in higher dimensions $(n>4)$ under some extra assumptions on the derivative of the curvature tensor. Namely, let $\left(M^{n}, g\right)$ be a complete Riemannian manifold which is conformally flat in a neighbourhood of infinity (with $n \geqslant 5)$. Assume now that it satisfies the Sobolev inequality

$$
\mu(M, g)\left(\int_{M} u^{\frac{2 n}{n-2}}\right)^{1-2 / n} \leqslant \int_{M}|d u|^{2}, \quad \forall u \in C_{0}^{\infty}(M)
$$

and that the Ricci curvature of $(M, g)$ satisfies

$$
\text { Ric } \in L^{n / 2}, \quad D R i c \in L^{n / 3} \cap L^{\frac{2 n}{n-2}}
$$

and that the scalar curvature satisfies for a $\delta \in] 0,1[$,

$$
\mathrm{Scal}_{g} \in L^{\frac{2 n}{n+2}} \cap L^{2(1+\delta)}, \quad D d \mathrm{Scal}_{g} \in L^{n / 4} \cap L^{2},
$$


and subeuclidean volume growth

$$
\operatorname{vol}_{g} B\left(x_{0}, r\right) \leqslant C\left(x_{0}\right) r^{n} \forall r \geqslant 1,
$$

then there is a bounded smooth function $f$ on $M$ such that $\left(M, e^{2 f} g\right)$ has a finite number of ends and each of them is asymptotically locally euclidean. Hence, Huber's theorem holds under these assumptions.

Proof of the remark. The proof of this last result follows basically the same path as the previous proofs, but is considerably more technical. It begins as the proof of Theorem 5.7 and the same argument leads to: $d u \in L^{\frac{2 n}{n-2}} \cap L^{2}$ and $D d u \in L^{2}$. This is not enough when $n \neq 4$, and it remains to show that $d u \in L^{n}$ and $D d u \in L^{n / 2}$. Moser iteration enters the picture here, applied to adequate inequations verified by $\alpha=d u$ and $D \alpha$ (in the notation of the proofs above). One then uses the following (Moser-type) analytical lemma:

Lemma 5.11. Let $(M, g)$ be a complete Riemannian manifold which satisfies the Sobolev inequality

$$
\mu(M, g)\left(\int_{M} u^{\frac{2 n}{n-2}}\right)^{1-2 / n} \leqslant \int_{M}|d u|^{2}, \quad \forall u \in C_{0}^{\infty}(M),
$$

and moreover we suppose that the volume growth of geodesic balls is sub-euclidean. Let $A \in L^{\tau}$ satisfying the inequation

$$
\Delta A \leqslant V A+f
$$

with $V \in L^{n / 2}$ and $f \in L^{p}$. If $p \leqslant \tau \leqslant \frac{n p}{n-2 p}$, then $A$ is in $L^{\frac{n p}{n-2 p}}$.

The lemma leads to the following direct corollary:

Corollary 5.12. Let $(M, g)$ be a complete Riemannian manifold. Under the same assumptions, if $A \in L^{\tau}$ satisfies the inequation

$$
\Delta A \leqslant V A+f
$$

with $V \in L^{n / 2}$ and $f \in L^{p_{-}} \cap L^{p_{+}}$, then, if $p_{-} \leqslant \tau \leqslant \frac{n p_{+}}{n-2 p_{+}}$, we get that $A \in L^{\frac{p_{+} n}{n-2 p_{+}}}$.

We postpone the proofs of these two results for a moment and notice that, once one has singled out an adequate inequation on $\alpha$ or $D \alpha$, the corollary, applied to both, finishes the proof of the remark.

As for the first step (inequation for $\alpha$ ) we have seen that the differential form $\alpha$ satisfies the equation

$$
\Delta \alpha+\operatorname{Ric} \alpha+c_{n} \operatorname{Scal}_{g} \alpha=-c_{n} u d \mathrm{Scal}_{g}+d \varphi .
$$

The Kato inequality implies that $A=|\alpha|$ satisfies the inequation:

$$
\Delta A \leqslant V A+f
$$


with $V=\operatorname{Ric}_{-}+c_{n}\left|\operatorname{Scal}_{g}\right|$ and $f=|d \varphi|+c_{n}\|u\|_{L^{\infty}} \mid d$ Scal $_{g} \mid$, with our hypothesis we have $V \in L^{n / 2}$ and $f \in L^{n / 3} \cap L^{2 n /(n-2)}$ and $A \in L^{2 n /(n-2)}$ so that we can employ the corollary and we obtain $A \in L^{n}$.

To pass on the case of $D \alpha$, we can differentiate again the inequality above and obtain

$$
\begin{aligned}
\Delta D \alpha+[D, \Delta] \alpha & +\left(\operatorname{Ric}_{g}+c_{n} \operatorname{Scal}_{g}\right) D \alpha= \\
& -(D \text { Ric }) \alpha-c_{n} d \operatorname{Scal}_{g} \otimes \alpha+D d \varphi+c_{n} \alpha \otimes d \operatorname{Scal}_{g}-c_{n} u D d \text { Scal }_{g}
\end{aligned}
$$

According to M. Le Couturier and G. Robert [21, Lemma 3.3.1],

$$
[D, \Delta] \alpha(X)=\frac{1}{2} D_{X} \operatorname{Ric} \alpha+R\left(D_{X} \alpha, .\right)+D_{\operatorname{Ric}(X)} \alpha .
$$

Since the manifold is conformally flat, the curvature tensor $R$ is bounded in terms of $\left|\operatorname{Ric}_{g}\right|$, so that the Kato inequality again yields that $B=|D \alpha|$ satisfies the inequation $\Delta B \leqslant W B+h$ where $W=b_{n}\left|\operatorname{Ric}_{g}\right|+c_{n}\left|\operatorname{Scal}_{g}\right|$ and

$$
h=C_{n}\left|D \operatorname{Ric}_{g}\right||\alpha|+|\alpha|\left|d \operatorname{Scal}_{g}\right|+c_{n}\|u\|_{L^{\infty}}\left|D d \operatorname{Scal}_{g}\right|+|D d \varphi| .
$$

Now our assumptions are: $h \in L^{2} \cap L^{n / 4}$, and $B \in L^{2}$, so that the Corollary above implies that $D d u \in L^{n / 2}$.

Proof of Lemma 5.11. This is shown in the following way, starting with the basic inequality (Lemma B.3 of [30]): if $\rho>1$ and $\psi \in C_{0}^{\infty}(M)$ then

$$
\int_{M}\left|d\left(\psi A^{\frac{\rho}{2}}\right)\right|^{2} \leqslant C(\rho) \int_{M}|d \psi|^{2} A^{\rho}+\psi^{2} A^{\rho-1} \Delta A
$$

We use our hypothesis on $A$, the Sobolev inequality and Hölder inequality in order to obtain:

$$
\begin{gathered}
\left(\mu-C(\rho)\|V\|_{L^{n / 2}(\operatorname{supp} \psi)}\right)\left\|\psi^{2} A^{\rho}\right\|_{L^{\frac{n}{n-2}}} \leqslant C(\rho)\|f\|_{L^{p}}\left(\int_{M} \psi^{\frac{2 p}{p-1}} A^{(\rho-1) \frac{p}{p_{1}}}\right)^{1-1 / p} \\
+C(\rho)\left(\int_{M}|d \psi|^{2 \lambda}\right)^{1 / \lambda}\left(\int_{M} A^{(\rho-1) \frac{p}{p-1}}\right)^{(1-1 / p)\left(\frac{\rho}{\rho-1}\right)}
\end{gathered}
$$

where $\lambda$ is defined by $\lambda^{-1}+(1-1 / p)\left(\frac{\rho}{\rho-1}\right)=1$. Assume now that $A$ lives in $L^{\tau_{0}}$ where $p \leqslant \tau_{0} \leqslant n p /(n-2 p)$. Let $\rho$ be defined by $\tau_{0}=(\rho-1) p /(p-1)$ and let $\tau_{1}=\frac{n}{n-2}\left(1+\frac{p-1}{p} \tau_{0}\right)$ so that $\lambda=\tau_{0} p /\left(\tau_{0}-p\right)$. Then choose $R_{0}$ such that

$$
C(\rho)\|V\|_{L^{n / 2}\left(M-B\left(x_{0}, R_{0}\right)\right)} \leqslant \mu / 2
$$

Using the sub-euclidean growth of the geodesic balls and applying the last inequality to a function $\psi=\psi_{R}$ such that $\operatorname{supp}\left(\psi_{R}\right) \subset B\left(x_{0}, 2 R\right)-B\left(x_{0}, R_{0}\right)$, $\psi_{R}=1$ on $B\left(x_{0}, R\right)-B\left(x_{0}, R_{0}+1\right), 0 \leqslant \psi_{R} \leqslant 1$ on $M$ and $\left|d \psi_{R}\right| \leqslant 2 / R$ on $B\left(x_{0}, 2 R\right)-B\left(x_{0}, R\right)$, we obtain

$$
\frac{\mu}{2}\|A\|_{L^{\tau_{1}}\left(B\left(x_{0}, R\right)-B\left(x_{0}, R_{0}+1\right)\right)} \leqslant C(\rho)\|A\|_{L^{\tau_{0}}}^{\rho-1}\|f\|_{L^{p}}+\left[C+C^{\prime} R^{n \frac{\tau_{0}-p}{\tau_{0} p}-2}\right]\|A\|_{L^{\tau_{0}}}^{\rho} .
$$


Now, since $\tau_{0} \leqslant p n /(n-2 p)$, we have $n \frac{\tau_{0}-p}{\tau_{0} p} \leqslant 2$. Letting $R$ tend to $\infty$ we get that $A \in L^{\tau_{1}}$. By iterating this procedure, we finally obtain that $A \in L^{r}$ for all $r \in[\tau, n p /(n-2 p)[$.

It remains to go up to the upper bound $n p /(n-2 p)$, and this is done in the following way: we apply again our first inequation with $\rho$ defined by

$$
\frac{n p}{n-2 p}=\rho \frac{n}{n-2}=(\rho-1) \frac{p}{p-1},
$$

and we choose $R_{0}, \psi$ similarly as above. It yields

$$
\frac{\mu}{2}\left\|\psi^{\frac{2}{\rho}} A\right\|_{L^{p n /(n-2 p)}}^{\rho} \leqslant C(\rho)\|f\|_{L^{p}}\left\|\psi^{\frac{2}{\rho}} A\right\|_{L^{p n /(n-2 p)}}^{\rho-1}+C(\rho) \int_{M}|d \psi|^{2} A^{\rho} .
$$

On the other hand, we have

$$
\int_{M}|d \psi|^{2} A^{\rho} \leqslant \int_{B\left(x_{0}, R_{0}+1\right)-B\left(x_{0}, R_{0}\right)}|d \psi|^{2} A^{\rho}+\frac{4}{R^{2}} \int_{B\left(x_{0}, 2 R\right)-B\left(x_{0}, R\right)} A^{\rho} .
$$

Since $\rho<p n /(n-2 p)$, we can choose a $\beta$ in $[\tau, n p /(n-2 p)[$ such that $\rho \leqslant \beta$. From the Hölder inequality and the volume growth of geodesic balls, we get

$$
\int_{B\left(x_{0}, 2 R\right)-B\left(x_{0}, R\right)}|d \psi|^{2} A^{\rho} \leqslant C R^{n(1-\rho / \beta)-2}\|A\|_{L^{\beta}}^{\rho} .
$$

As $\beta<n p /(n-2 p)$, we have $n(1-\rho / \beta)<2$, and finally $A \in L^{n p /(n-2 p)}$ when $R$ goes to infinity.

The case of the sphere. The results of the current section can also be applied to the case of a domain in the 4-dimensional sphere and we get a strengthening of Corollary 2.6 for this precise dimension. As already noticed in section 2, the Sobolev inequality is automatically obtained in this case, hence the following result (analogous results may be obtained for any other conformally flat compact manifold with positive Yamabe invariant):

Corollary 5.13. Let $\Omega$ be a domain of the sphere $\left(\mathbf{S}^{4}, g_{0}\right)$ endowed with a complete metric $g$ conformal to $g_{0}$. Assume moreover

$$
\left.\operatorname{Ric}_{g} \in L^{2}(\Omega, g) \quad \text { and } \quad \operatorname{Scal}_{g} \in L^{\frac{4}{3}}(\Omega, g) \cap L^{2(1+\delta)}(\Omega, g) \quad \text { for some } \delta \in\right] 0,1[\text {. }
$$

Then there is a finite set $\left\{p_{1}, \ldots, p_{k}\right\}$ such that $\Omega=\mathbf{S}^{4}-\left\{p_{1}, \ldots, p_{k}\right\}$.

This may be compared with S. Y. A. Chang, J. Qing and P. Yang's proof [8] that any domain in the 4-sphere with a complete metric conformal to the round one, with Ricci curvature bounded from below, bounded "Q-curvature" (the integral of the local curvature expression that gives the Gauss-Bonnet-Chern integrand) and uniformly positive scalar curvature is the sphere minus a finite number of points.

Acknowledgements. The authors would like to thank Daniel Grieser for a useful discussion, Dominique Hulin, Jacques Lafontaine and François Laudenbach for their precious remarks on earlier versions of this paper. 


\section{References}

[1] U. Abresch, Lower curvature bounds, Toponogov's theorem and bounded topology, I, Ann. Scient. Ec. Norm. Sup. 18 (1985), 651-670.

[2] M. T. Anderson, Convergence and rigidity of manifolds under Ricci curvature bounds, Invent. Math. 102 (1990), 429-445.

[3] M. T. Anderson and J. Cheeger, $C^{\alpha}$ compactness for manifolds with Ricci curvature and injectivity radius bounded below, J. Diff. Geom. 35 (1992), 265-281.

[4] S. Bando, A. Kasue and H. Nakajima, On a construction of coordinates at infinity on manifolds with fast curvature decay and maximal volume growth, Invent. Math. 97 (1989), 313-349.

[5] J. P. Bourguignon, Les variétés de dimension 4 à signature non-nulle et dont la courbure est harmonique sont d'Einstein, Invent. math. 63 (1981), 263-286.

[6] D. M. J. Calderbank, P. Gauduchon and M. Herzlich, Refined Kato inequalities and conformal weights in Riemannian geometry, J. Funct. Anal. 173 (2000), 214-255.

[7] G. Carron, Inégalités de Faber-Krahn et conséquences, Actes de la table ronde de géométrie différentielle en l'honneur de M. Berger, Séminaires et Congrès, vol. 1, Soc. Math. France, 1994, pp. 205-232.

[8] G. Carron, Une suite exacte en $L^{2}$-cohomologie, Duke Math. J. 95 (1998), 343-372.

[8] S. Y. A. Chang, J. Qing and P. C. Yang, Compactification of a class of conformally flat 4-manifold, Invent. Math. 142 (2000), 65-93.

[10] J. Cheeger, Finiteness theorems for Riemannian manifolds, Amer. J. Math. 92 (1970), 61-74.

[11] J. Cheeger, M. Gromov and M. Taylor, Finite propagation speed, kernel estimates for functions of the Laplace operator and the geometry of complete Riemannian manifolds, $J$. Diff. Geom. 17 (1982), 15-53.

[12] T. Coulhon, Inégalités de Gagliardo-Nirenberg pour les semi-groupes d'opérateurs et applications, Potential Analysis 1 (1992), 343-353.

[13] P. Delanoë, Generalized stereographic projections with prescribed scalar curvature, Contemporary Math. 127 (1992), 17-25.

[14] D. Gilbarg and N. S. Trudinger, Elliptic Partial Differential Equations of Second Order, Grundlehr. Math. Wiss., vol. 224, Springer, Berlin, 1977.

[15] R. Greene, P. Petersen and S. Zhu, Riemannian manifolds of faster-than-quadratic curvature decay, Int. Math. Res. Notices 9 (1994), 363-377.

[16] E. Hebey and M. Herzlich, Harmonic coordinates, harmonic radius and convergence of Riemannian manifolds, Rend. Mat. Appl. (Roma) 17 (1997), 569-605.

[17] M. Herzlich, Compactification conforme des variétés asymptotiquement plates, Bull. Soc. Math. France 125 (1997), 55-92.

[18] A. Huber, On subharmonic functions and differential geometry in the large, Comment. Math. Helv. 32 (1957), 13-72.

[19] W. Hurewicz and H. Wallmann, Dimension theory, Princeton. Univ. Press, Princeton, 1948.

[20] A. Kasue, A compactification of a manifold with asymptotically nonnegative curvature, Ann. Scient. Ec. Norm. Sup. Paris 21 (1988), 593-622.

[21] M. Le Couturier and G. Robert, $L^{p}$-pinching and the geometry of compact Riemannian manifolds, Comment. Math. Helv. 69 (1994), 249-271.

[22] J. Lee and T. H. Parker, The Yamabe problem, Bull. Amer. Math. Soc. 17 (1987), 37-91.

[23] P. Petersen, Riemannian geometry, Grad. Text. Math., vol. 171, Springer, Heidelberg, 1998.

[24] R. Schoen and S. T. Yau, Conformally flat manifolds, Kleinian groups and scalar curvature, Invent. Math. 92 (1988), 47-71.

[25] J.-P. Sha and Z. Shen, Complete manifolds with nonnegative Ricci curvature and quadratically nonnegatively curved infinity, Amer. J. Math. 119 (1997), 1399-1404.

[26] J.-P. Sha and D.-G. Yang, Examples of manifolds of positive Ricci curvature, J. Diff. Geom. 29 (1989), 95-103. 
[27] K. Uhlenbeck, Removable singularities for Yang-Mills fields, Commun. Math. Phys. 83 (1982), 11-30.

[28] S. Unnebrink, Asymptotically flat four-manifolds, Diff. Geom. Appl. 6 (1996), 271-274.

[29] N. Varopoulos, Hardy-Littlewood theory for semigroups, J. Funct. Anal. 63 (1985), 240260.

[30] D. Yang, Convergence of Riemannian manifolds with integral bounds on curvature, II, Ann. Scient. Ec. Norm. Sup. Paris 25 (1992), 179-199.

[31] S. Zhu, A volume comparison theorem for manifolds with asymptotically nonnegative curvature and its application, Amer. J. Math. 116 (1994), 669-682.

Gilles Carron

Département de Mathématiques

CNRS UMR 6629

Université de Nantes

Case postale 92208

F-44322 Nantes-Cedex 3

France

e-mail: Gilles.Carron@math.univ-nantes.fr
Marc Herzlich

Département de Mathématiques

CNRS UMR 5030

Université Montpellier II

case 51

F-34095 Montpellier-Cedex 5

France

e-mail: herzlich@math.univ-montp2.fr

(Received: April 14, 2000; revised version: March 20, 2001)

(17) To access this journal online:

(20) http://www.birkhauser.ch 